\title{
Cálculo de la magnitud límite estelar (MALE) en Tegucigalpa
}

\author{
Roberto-Ernesto Shöngarth-Carías ${ }^{1, *}$, Ricardo-Antonio Pastrana-Sánchez ${ }^{2,+}$ \\ ${ }^{1}$ Máster, Western International School of Honduras \\ ${ }^{2}$ Máster, Departamento de Astronomía y Astrofísica de la UNAH \\ DOI: https://doi.org/10.5377/ce.v11i2.8678
}

\begin{abstract}
RESUMEN
El trabajo de investigación consistió en la recopilación de la Magnitud Límite Estelar (MALE) de Tegucigalpa, además de un dato adicional de Talanga, a fin de tener una referencia rural de comparación. Las magnitudes límites para la ciudad de Tegucigalpa desde diferentes ubicaciones arrojaron resultados de 2.98 (baja debido a contaminación lumínica en la zona), 3.56, 3.58 y 3.76. El promedio de la magnitud observada fue de 3.53 con una desviación estándar de 0.41. La mediana es de 3.56. Estos resultados nos indican que el cielo de Tegucigalpa es de cuarta magnitud en la escala de Bortle. Las condiciones en Talanga, cielo rural, mejoran hasta 4.68, lo que podría estimarse de una quinta magnitud, considerados cielos de transición entre periurbano y urbano. Las condiciones meteorológicas impidieron crear un registro estacional. Solo durante los meses de noviembre y enero se tuvieron condiciones de observación de buena calidad. En marzo se observa un cielo despejado, pero con condiciones de bruma debido a la quema de bosques que rodean la ciudad.
\end{abstract}

Palabras clave: magnitud, brillo del cielo, constelación, contaminación lumínica, polución lumínica, MALE.

\begin{abstract}
The research work is about the compilation of the Stellar Limit Magnitude (MALE) of Tegucigalpa, as well as an additional data from Talanga, in order to obtain a rural reference for comparison. The Tegucigalpa's limit magnitudes from different locations are 2.98 (low value due to light pollution in those places), 3.56, 3.58 and 3.76. The magnitude average is 3.53 with a standard deviation of 0.41 . The median is 3.56. These results indicate that the Tegucigalpa's sky is of fourth magnitude in the Bortle scale. Conditions in Talanga, a rural sky, improve up to 4.68 , that can be estimated of fifth magnitude, considered transitional skies between peri-urban and urban. Meteorological conditions prevented to create a seasonal record. Only in November and January were obtained good quality observational conditions. A good sky was observed in March, but with fog due to forest fire around the city.
\end{abstract}

Keywords: magnitude, brightness of the sky, constellation, light pollution, MALE.

\section{Introducción}

La medición de la Magnitud Límite Estelar (MALE) tiene como objetivo la medición de la calidad de cielo a través de la estimación de la magnitud hasta la cual se puede observar a simple vista, en relación a la sexta

*robertoschongarth@gmail.com

${ }^{\dagger}$ r_pastrana@hotmail.com 
magnitud de las mediciones que Hiparco realizaró en el siglo II a.C. La magnitud límite estelar, es la magnitud aparente de la estrella más débil cerca del cenit (Mallén, 2010) que el observador pueda detectar a simple vista. Conocer su valor permite estimar la transparencia del cielo, la cual se ve afectada por la polución lumínica que atenúa el brillo de los objetos celestes. La MALE permite conocer el cielo con el que se cuenta para la observación astronómica. El Departamento de Astronomía y Astrofísica (DAAF) cuenta con investigaciones científicas sobre la caracterización del cielo del Observatorio Astronómico Centroamericano de Suyapa (OACS) como el Seeing y el Brillo del Fondo del Cielo (BFC). El cálculo de la MALE complementaría la valoración de las condiciones de observación astronómica del observatorio de la UNAH. El valor calculado de la MALE permite advertir a los observadores cual es la calidad de observación nocturna del cielo de Tegucigalpa. Adicionalmente los resultados al término de la investigación indicaron la evolución de la contaminación lumínica de la ciudad. La metodología de observación y registro de datos, se basa en los pasos sugeridos por Globe at Night ${ }^{1}$. Nuestros datos fueron obtenidos en diferentes puntos de la ciudad y uno de zona rural para efecto de comparación. El trabajo justifica la razón para efectuar la investigación y los motivos por los que es importante registrar las condiciones del cielo de una determinada ubicación.

\subsection{Importancia de MALE}

La transparencia del cielo ha cambiado mucho desde que Hiparco de Nicea en el año 130 a.C realizara observaciones astronómicas a simple vista. Él categorizó unas mil estrellas basándose en el brillo de éstas, a las cuales asignó un número al que llamó magnitud de acuerdo a qué tan brillantes se veían, así las estrellas más brillantes eran de $1^{a}$ magnitud y las casi imperceptibles de $6^{a}$ magnitud. El desarrollo tecnológico e industrial del ser humano ha modificado en gran medida la posibilidad de observar objetos tenues en el cielo. Esto se debe principalmente a dos razones: la primera es la contaminación de la atmósfera por las fuertes emisiones de gases industriales y en segundo lugar está la contaminación lumínica ocasionada por el alumbrado público y la publicidad luminosa que se presenta en gran escala en las ciudades. Los observatorios astronómicos se ven afectados de manera directa por este tipo de contaminación ya que la luz de las ciudades es emitida en gran cantidad hacia el cielo, la radiación es reflejada y difuminada por los gases y partículas presentes en aire, provocando una disminución en la capacidad de observación de objetos celestes en el cielo nocturno. Saber cómo afecta la emisión de radiación en exceso causada por la polución lumínica de Tegucigalpa y qué sugerencias ofrecer para aplicar los correctivos al respecto dependen de las mediciones de la MALE de la ciudad. Por lo tanto, la MALE nos indica cual es el nivel de transparencia del cielo nocturno del sitio de observación, brindándonos una buena estimación de la atenuación que sufre la luz proveniente de los astros debido a la magnitud del cielo de fondo.

\section{Metodología}

La investigación posee un enfoque cuantitativo. El estudio es longitudinal, pues se mide la evolución de la variable dependiente a través de mediciones realizadas en diferentes instantes. El diseño es no experimental con un alcance descriptivo. No hay manipulación de variables y el estudio se limita a una descripción de los fenómenos de variación de la magnitud estelar. El cálculo de la Magnitud Límite Estelar se realizó siguiendo el "Método del conteo de estrellas" sugerido por International Meteor Organization el cual consiste en determinar la magnitud de la estrella más débil cercana al cenit que el observador pueda detectar a ojo desnudo. El método permitió evaluar la calidad del cielo nocturno del sitio de observación en forma estadística debido a la participación de varios observadores a la vez. La toma de los datos se hizo mediante la observación

\footnotetext{
${ }^{1}$ El proyecto Globe at Night ha sido una iniciativa mundial (Observatory, 2016) que motiva a los diferentes países a tratar de conocer las características del cielo a través de la medición de la Magnitud Límite Estelar.
} 
de diferentes zonas del cielo previamente establecidas (Mike Hankey, 2019). El punto de observación, fue el Observatorio Astronómico Centroamericano de Suyapa (OACS). El cómputo estelar se hizo de forma simultánea entre todos los observadores evitando que la masa de aire sea un factor incidente en la observación. Para la recolección de los datos fue necesaria la organización de un grupo de observación, se logró contar con la contribución de cuatro observaciones realizadas por voluntarios. Las efemérides de las zonas de cielo a contar, tomando en cuenta la fecha de observación UT (Universal time) y LT (Local time), pronóstico del clima y distancia cenital. Las alturas en las cuales se realizaron las mediciones son: $z \leq 40^{\circ}$. Las predicciones de las posiciones astronómicas de las zonas de observación de cielo se hicieron mediante el servicio en línea que presta el Observatorio Roque de los Muchachos que permite: 1. representar la altura en función del tiempo para una noche especial, 2. trazar la trayectoria de los objetos a través del cielo, 3. dibujar los cambios de altura para más de un año, consiguiendo la mejor fecha de observación para cada objeto. Este servicio se encuentra disponible en la dirección siguiente:http://catserver.ing.iac.es/staralt/index.php

Con el fin de obtener una mejor estadística, se pretendió que los conteos de estrellas se realizaran de forma simultánea para una misma zona del cielo. Esta actividad no fue posible llevarla a cabo, debido a que las condiciones de nubosidad fueron muy variables para cada observador. El cálculo de la MALE se hizo tomando en cuenta las cartas de cielo y tablas de magnitud límite proporcionadas por la International Meteor Organization. Se elaboraron tablas que continen las condiciones de clima (nubosidad, humedad y fase lunar) en cada una de las jornadas de medición.

Con los datos derivados de las observaciones astronómicas para conteo de estrellas según cada zona de cielo se realizaron las siguientes operaciones: 1. cálculo la MALE por zona de medición y observador, 2. cálculo de la media para cada zona de estudio, 3. Calculó de la media por cada observador, 4. determinación de la magnitud límite puntual. Lo anterior permitió indicar las zonas que presentan mejores condiciones de observación. Inicialmente hay que dejar que los ojos se adapten durante al menos 30 minutos para después comenzar con el conteo de estrellas que revela hasta qué magnitud límite ha sido posible observar (Makela, 2016). Para asegurar observaciones en condiciones óptimas de oscuridad de cielo, se debió esperar la hora del crepúsculo astronómico, es decir cuando la altura del Sol es de $18^{\circ}$ bajo el horizonte. Como referencia, las horas de los crepúsculos astronómicos en los solsticios, que son los momentos del año que marcan las posiciones extremas del Sol durante el año, pueden ser calculadas, tomando como referencia la hora de salida o puesta del Sol como:

$$
\begin{gathered}
\Delta H=\cos ^{-1}\left[\frac{\sin \left(-18^{\circ}\right)-\sin \left(14^{\circ}\right) \sin \left(23^{\circ} 26^{\prime}\right)}{\cos \left(14^{\circ}\right) \cos \left(23^{\circ} 26^{\prime}\right)}\right]-\cos ^{-1}\left[\frac{\sin \left(-18^{\circ}\right)-\sin \left(14^{\circ}\right) \sin \left(23^{\circ} 26^{\prime}\right)}{\cos \left(14^{\circ}\right) \cos \left(23^{\circ} 26^{\prime}\right)}\right] \\
\Delta H=1 \mathrm{~h} 9 \mathrm{~m} 56 \mathrm{~s} \text { (Solsticio de verano) } \\
\Delta H=\cos ^{-1}\left[\frac{\sin \left(-18^{\circ}\right)-\sin \left(14^{\circ}\right) \sin \left(-23^{\circ} 26^{\prime}\right)}{\cos \left(14^{\circ}\right) \cos \left(23^{\circ} 26^{\prime}\right)}\right]-\cos ^{-1}\left[\frac{\sin \left(-18^{\circ}\right)-\sin \left(14^{\circ}\right) \sin \left(-23^{\circ} 26^{\prime}\right)}{\cos \left(14^{\circ}\right) \cos \left(23^{\circ} 26^{\prime}\right)}\right] \\
\Delta H=1 \mathrm{~h} 16 \mathrm{~m} 35 \mathrm{~s} \text { (Solsticio de invierno) }
\end{gathered}
$$

Hay que considerar que en el solsticio de verano las horas de salida y puesta del Sol son a las 5:23 y las 18:18 respectivamente, mientras que durante el solsticio de invierno estas horas son a las 6:09 y 17:24. Al necesitar completa oscuridad, no se recomienda hacer observaciones de cielo profundo más tarde que las 4 de la mañana o más temprano que las 19:30 de la noche en función de la estación del año. 
Hay dos factores predecibles y uno que no lo es, para poder planear las observaciones. El primero consiste precisamente en las horas de observación entre los crepúsculos astronómicos, y el segundo es el de la presencia o ausencia de Luna, ya que la Luna obstruye la visibilidad del cielo profundo y las estrellas se observan con un brillo atenuado. El factor no predecible es la nubosidad. Durante las observaciones se llevó a cabo una anotación acerca de las condiciones meteorológicas y posteriormente se verifico con la información del Servicio Nacional de Meteorología para corroborar cómo estuvieron las condiciones durante todos y cada uno de los días del año.

La tabla 1 contiene las efemérides previas a la observación. Por ejemplo, el 20 de enero se daba la posibilidad de observar el cielo desde el crepúsculo astronómico hasta la una de la mañana, hora aproximada en que sale la Luna. Si se desea hacer una observación ese día, por ejemplo, entre 10 y 11 de la noche, las zonas de cielo a observar serán las 4, 8 y 17 según el Atlas Brno (Znojil, 1988).

Tabla 1: Zonas de observación de cielo en función de la hora, la fecha y la presencia de Luna marcada en color azul

\begin{tabular}{|c|c|c|c|c|c|c|c|c|c|c|c|c|c|c|c|c|c|c|c|c|c|c|c|c|c|c|c|c|}
\hline Fecha & \multicolumn{3}{|c|}{$19-20 \mathrm{~h}$} & \multicolumn{3}{|c|}{$20-21 \mathrm{~h}$} & \multicolumn{3}{|c|}{$21-22 \mathrm{~h}$} & \multicolumn{3}{|c|}{$22-23 \mathrm{~h}$} & \multicolumn{3}{|c|}{$23-00 \mathrm{~h}$} & \multicolumn{3}{|c|}{$00-01 \mathrm{~h}$} & \multicolumn{3}{|c|}{$01-02 \mathrm{~h}$} & \multicolumn{3}{|c|}{$02-03 \mathrm{~h}$} & \multicolumn{3}{|c|}{ 03-04 h } & Fecha \\
\hline 17-01-17 & 2 & 8 & 22 & 2 & 8 & 22 & 4 & 8 & 22 & 4 & 8 & 17 & 4 & 8 & 17 & 3 & 4 & 9 & 3 & \begin{tabular}{|l|}
4 \\
\end{tabular} & 9 & 9 & \begin{tabular}{|l|}
10 \\
\end{tabular} & 23 & 9 & 10 & 23 & 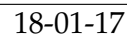 \\
\hline 18-01-17 & 2 & & 22 & 2 & 8 & 22 & 4 & & 22 & 4 & & 17 & 4 & 8 & 17 & . & 4 & 9 & 3 & 4 & 9 & 9 & 10 & 23 & 9 & 10 & 23 & 19-01-17 \\
\hline 19-01-17 & 2 & & 22 & 2 & 8 & 22 & 4 & 8 & 22 & 4 & 8 & 17 & 4 & 8 & 17 & 3 & 4 & 9 & 3 & 4 & 9 & 9 & 10 & 23 & 9 & 10 & 23 & $20-01-17$ \\
\hline $20-01-17$ & 2 & $\varepsilon$ & 22 & 2 & 8 & 22 & 4 & 8 & 22 & 4 & $\varepsilon$ & 17 & 4 & 8 & 17 & 3 & 4 & 9 & 3 & 2 & 5 & 9 & 10 & 23 & 9 & 10 & 23 & $21-01-17$ \\
\hline 21-01-17 & & $\varepsilon$ & 22 & 2 & 8 & 22 & 4 & 8 & 22 & 4 & 8 & 17 & 4 & 8 & 17 & & 4 & 9 & 3 & 4 & 9 & 9 & 10 & 23 & 9 & 10 & 23 & $22-01-17$ \\
\hline $22-01-17$ & & 8 & 22 & 2 & 8 & 22 & 4 & $\varepsilon$ & 22 & 4 & 8 & 17 & 4 & 8 & 17 & & 4 & 9 & 3 & 4 & 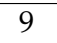 & 9 & 10 & 23 & y & 10 & 23 & $23-01-17$ \\
\hline $23-01-17$ & & 8 & 22 & 2 & 8 & 22 & 4 & & 22 & 4 & 8 & 17 & 4 & 8 & 17 & 3 & 4 & 9 & 3 & 4 & 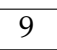 & 9 & 10 & 23 & 9 & 10 & 23 & 24-01-17 \\
\hline $24-01-17$ & 2 & $\varepsilon$ & 22 & 2 & 8 & 22 & 4 & 8 & 22 & 4 & 8 & 17 & 4 & 8 & 17 & 3 & 4 & 9 & 3 & 4 & 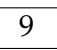 & 9 & 10 & 23 & y & 10 & 23 & $25-01-17$ \\
\hline $25-01-17$ & 2 & & 22 & 2 & 8 & 22 & 4 & 8 & 17 & 4 & 8 & 17 & 4 & 8 & 9 & 3 & 4 & 9 & 3 & 4 & 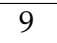 & 9 & 10 & 23 & $y$ & 10 & 23 & $26-01-17$ \\
\hline 26-01-17 & 2 & 8 & 22 & 8 & 17 & 22 & 4 & 8 & 17 & 4 & 8 & 17 & 4 & 8 & 9 & 3 & 4 & 9 & 3 & 4 & 9 & 9 & 10 & 23 & $y$ & 10 & 23 & 27-01-17 \\
\hline 27-01-17 & 2 & 8 & 22 & 4 & 8 & 22 & 4 & 8 & 17 & 4 & 8 & 17 & 4 & 8 & 9 & 3 & 4 & 9 & 3 & 4 & 9 & 9 & 10 & 23 & y & 10 & 23 & $28-01-17$ \\
\hline 28-01-17 & 2 & 8 & 22 & 4 & 8 & 22 & 4 & 8 & 17 & 4 & 8 & 17 & 4 & 8 & 9 & 3 & 4 & 9 & 3 & 4 & 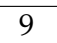 & 9 & 10 & 23 & 9 & 10 & 23 & 29-01-17 \\
\hline 29-01-17 & 2 & 8 & 22 & 4 & 8 & 22 & 4 & 8 & 17 & 4 & 8 & 17 & 4 & 8 & 9 & 3 & 4 & 9 & 3 & 4 & 9 & 9 & 10 & 23 & 9 & 10 & 23 & 30-01-17 \\
\hline $30-01-17$ & 2 & 8 & 22 & 4 & 8 & 22 & 4 & 8 & 17 & 4 & 8 & 17 & & 8 & 17 & & 4 & 9 & 3 & 9 & 23 & 9 & 10 & 23 & 9 & 10 & 23 & 31-01-17 \\
\hline 31-01-17 & 2 & 8 & 22 & 4 & 8 & 22 & 4 & 8 & 17 & 4 & 8 & 17 & 4 & 8 & 17 & 3 & 4 & 9 & 3 & 9 & 23 & 9 & 10 & 23 & 9 & 10 & 16 & 01-02-17 \\
\hline $01-02-17$ & 2 & 8 & 22 & 4 & 8 & 22 & 4 & 8 & 17 & 4 & 8 & 17 & 3 & 4 & 9 & 3 & 4 & 9 & 3 & 9 & 10 & 9 & 10 & 23 & 9 & 10 & 11 & $02-02-17$ \\
\hline $02-02-17$ & 2 & 8 & 22 & 4 & 8 & 22 & 4 & 8 & 17 & 4 & 8 & 17 & 3 & 4 & $\overline{9}$ & 3 & 4 & 9 & $\overline{9}$ & 10 & 23 & 9 & 10 & 23 & 9 & 10 & 11 & $03-02-17$ \\
\hline 03-02-17 & 2 & 8 & 22 & 4 & 8 & 22 & 4 & 8 & 17 & 4 & 8 & 17 & 3 & 4 & 9 & 3 & 4 & 9 & 9 & 10 & 23 & 9 & 10 & 23 & 9 & 10 & 11 & $04-02-17$ \\
\hline $04-02-17$ & 2 & $\varepsilon$ & 22 & 4 & 8 & 22 & 4 & 8 & 17 & 4 & 8 & 17 & 3 & 4 & 9 & 3 & 4 & 9 & 9 & 10 & 23 & 9 & 10 & 23 & 9 & 10 & 11 & $05-02-17$ \\
\hline 05-02-17 & 2 & 8 & 22 & 4 & 8 & 22 & 4 & 8 & 17 & 4 & 8 & 17 & & 4 & s & 3 & 4 & 9 & 9 & 10 & 23 & 9 & 10 & 23 & 9 & 10 & 11 & $06-02-17$ \\
\hline 06-02-17 & 2 & 8 & 22 & 4 & 8 & 22 & 4 & 8 & 17 & 4 & 8 & 17 & 3 & 4 & 9 & 3 & 4 & 9 & 9 & 10 & 2 & 9 & 10 & 23 & 9 & 10 & 11 & 07-02-17 \\
\hline $07-02-17$ & 2 & 8 & 22 & 4 & 8 & 22 & 4 & 8 & 17 & 4 & 8 & 17 & 3 & 4 & 9 & 3 & 4 & 9 & 9 & 10 & 23 & 9 & 10 & 23 & 9 & 10 & 11 & $08-02-17$ \\
\hline
\end{tabular}

\section{Resultados}

La trascendencia de los datos meteorológicos durante el transcurso del trabajo. Se decidió agregar un análisis de las condiciones de nubosidad en el transcurso del año, pues éstas, combinadas con la aparición de la Luna, dejan una estadística elocuente acerca de las condiciones de observación en la ciudad de Tegucigalpa. Los datos meteorológicos recopilados fueron tomados de la página de http://ogimet.com/, bajo la recomendación de Erick Martínez, de la Sección de Climatología Aeronáutica de la Agencia Hondureña de Aeronáutica Civil. Los datos aparecen tabulados en intervalos de tres horas e incluyen nubosidad, precipitación, temperatura, y otros.

La nubosidad es medida en octavas, que indica cuántas octavas partes de cielo se encontraban cubiertas en el momento de la toma de datos. La tabla 2 resume las frecuencias de octavas de nubosidad, durante el año que 
transcurre entre el 26 de noviembre de 2016 y el 25 de noviembre de 2017. Para ello solamente se ha incluido la toma de datos efectuada en momentos de ausencia de luna, por lo que hay una muestra de 838 puntos en el año en los cuales no hay presencia de luna. Se observa en la tabla 2 que, para las horas 18, 21, 00,03 y 06 la nubosidad más frecuente en cada una de ellas corresponde a las 7 octavas, o sea un cielo casi completamente cubierto. En la parte inferior de la tabla se agrega la media de octavas durante cada una de las horas, donde se observa que la media de octavas oscila entre 5.0190 y 5.7778 , es decir un cielo nublado más de la mitad del año.

Tabla 2: Frecuencia anual de octavas de nubosidad en días sin Luna, para algunas horas

\begin{tabular}{cccccc}
\hline Octavas & $18 \mathrm{~h}$ & $21 \mathrm{~h}$ & $00 \mathrm{~h}$ & $03 \mathrm{~h}$ & $06 \mathrm{~h}$ \\
\hline 0 & 0 & 1 & 2 & 6 & 0 \\
1 & 8 & 19 & 21 & 14 & 12 \\
2 & 9 & 13 & 10 & 13 & 11 \\
3 & 9 & 12 & 10 & 7 & 3 \\
4 & 14 & 11 & 15 & 10 & 11 \\
5 & 16 & 15 & 9 & 12 & 17 \\
6 & 13 & 19 & 24 & 18 & 14 \\
7 & 95 & 73 & 63 & 84 & 101 \\
8 & 7 & 5 & 4 & 4 & 4 \\
\hline Media & 5.7778 & 5.1488 & 5.0190 & 5.2917 & 5.7514 \\
Válidos & 171 & 168 & 158 & 168 & 173 \\
\hline
\end{tabular}

La tabla 3 muestra cuáles son las épocas ideales para hacer observaciones de cielo desde Tegucigalpa. Analizando la tabla 3, se muestran resaltadas en rojo aquellas celdas en que los porcentajes son más altos. Evidentemente en cada mes vuelven a ser más frecuentes los cielos de siete octavas, aunque lo más relevante es observar cuáles meses permiten observaciones de cielo. En el año que se tomó de muestra se observan los meses de noviembre, enero y marzo, aunque los verdaderamente válidos son los dos primeros pues durante el mes de marzo comienza la temporada de quemas en el país, por lo que en el cielo se distingue una continua bruma. A partir de allí y hasta el mes de octubre, los cielos poseen una nubosidad constante y hace muy difícil encontrar condiciones óptimas para la observación.

Tabla 3: Porcentaje de octavas de nubosidad en días sin Luna, por mes

\begin{tabular}{ccccccccccccc}
\hline Octavas & Enero & Febrero & Marzo & Abril & Mayo & Junio & Julio & Agosto & Septiembre & Octubre & Noviembre & Diciembre \\
\hline 0 & 5.88 & 0.00 & 0.00 & 1.45 & 0.00 & 0.00 & 0.00 & 0.00 & 0.00 & 0.00 & 2.53 & 1.37 \\
1 & 27.94 & 4.11 & 17.11 & 7.25 & 6.06 & 0.00 & 0.00 & 4.11 & 0.00 & 0.00 & 15.19 \\
2 & 23.53 & 4.11 & 14.47 & 8.70 & 0.00 & 1.52 & 1.49 & 4.11 & 1.49 & 1.41 & 5.06 \\
3 & 2.94 & 0.00 & 11.84 & 7.25 & 0.00 & 4.55 & 2.99 & 0.00 & 0.00 & 1.41 & 6.33 & 10.96 \\
4 & 11.76 & 6.85 & 7.89 & 10.14 & 4.55 & 6.06 & 5.97 & 6.85 & 1.49 & 5.63 & 13.92 & 5.48 \\
5 & 7.35 & 8.22 & 17.11 & 7.25 & 1.52 & 7.58 & 7.46 & 8.22 & 2.99 & 2.82 & 11.39 & 4.11 \\
6 & 4.41 & 21.92 & 5.26 & 10.14 & 13.64 & 7.58 & 11.94 & 21.92 & 7.46 & 11.27 & 6.33 & 16.44 \\
7 & 16.18 & 54.79 & 26.32 & 46.38 & 68.18 & 60.61 & 67.16 & 54.79 & 80.60 & 71.83 & 39.24 & 49.32 \\
8 & 0.00 & 0.00 & 0.00 & 1.45 & 6.06 & 12.12 & 2.99 & 0.00 & 5.97 & 5.63 & 0.00 & 0.00 \\
\hline
\end{tabular}

Las figuras 1 y 2, muestran los puntos en los cuales fueron recopilados los datos. En la figura 1 se tiene un campo amplio para incluir la observación asociada a Talanga. En la figura 2 se hace un acercamiento a Tegucigalpa para mostrar desde qué lugares se hicieron las observaciones.

Es evidente la diferencia entre el cielo urbano de Tegucigalpa y el rural de Talanga, por lo que la posibilidad 
de observar el cielo profundo aumenta. La magnitud registrada en Talanga fue de 4.68, por lo que pudiera afirmarse que este sitio posee un cielo que permite observar a una quinta magnitud.

El cielo de Tegucigalpa presenta diferentes problemas. Por ejemplo, observando el sitio marcado con 2.98, hacia el sur, presenta una magnitud no muy significativa pues, a pesar de estar en las afueras de la ciudad, es afectado por contaminación lumínica.

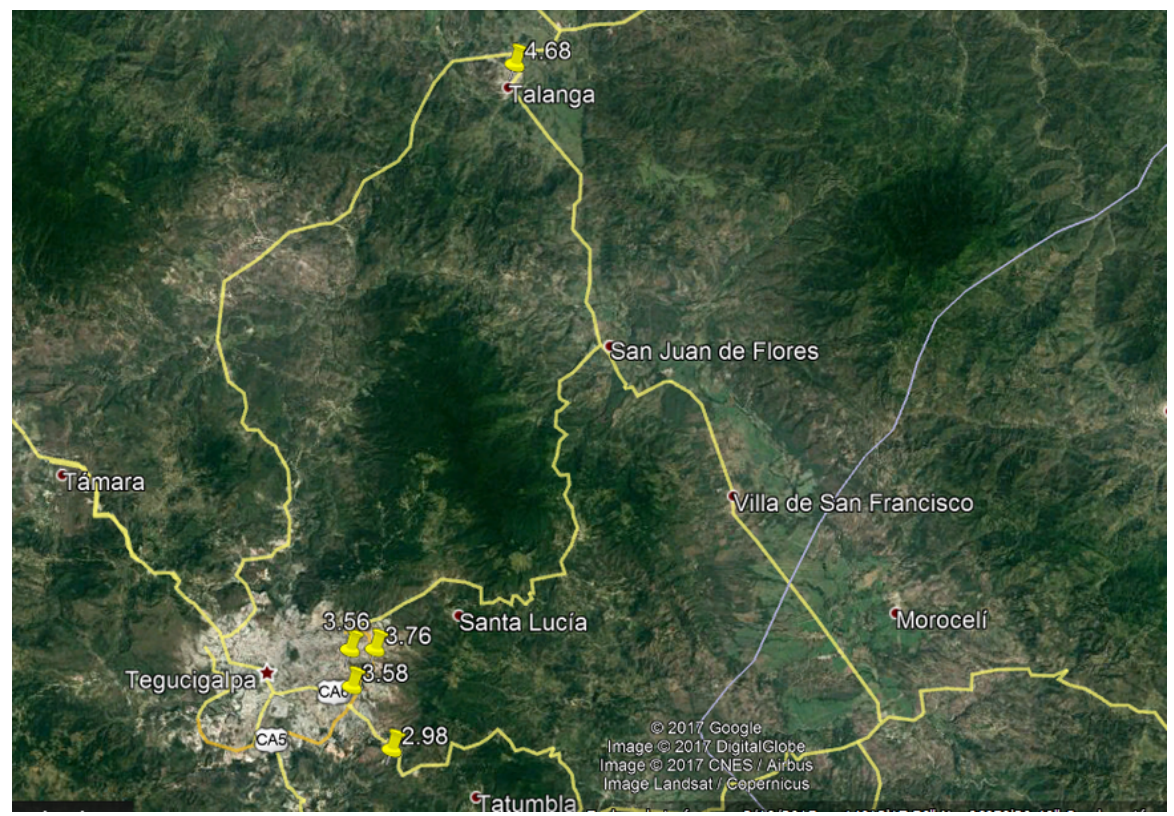

Figura 1: Magnitudes medidas en Tegucigalpa y Talanga. Imagen recuperada de Google Earth.

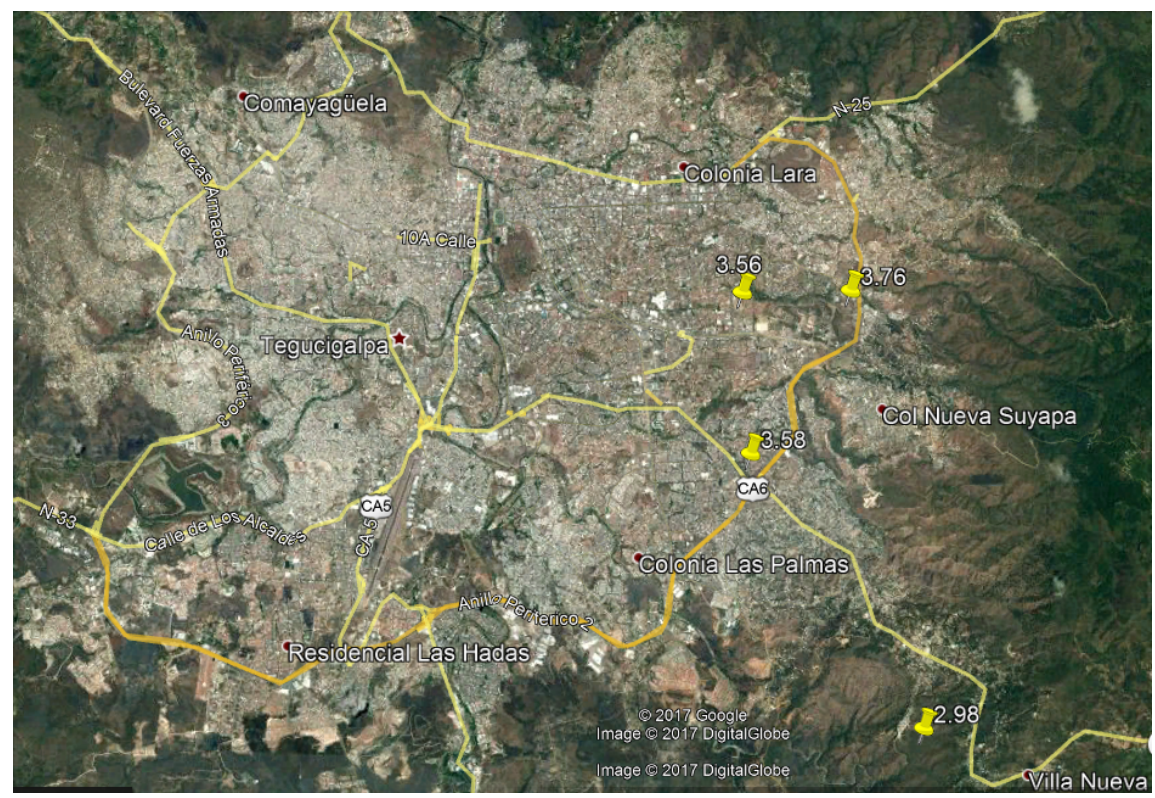

Figura 2: Acercamiento a Tegucigalpa. Magnitud límite por cada ubicación. Imagen recuperada de Google Earth.

Los otros tres puntos muestran magnitudes parecidas $(3.56,3.58$ y 3.76$)$ y según las observaciones, posiblemente son los cielos más oscuros que puedan tenerse dentro de la ciudad. La contaminación lumínica dentro 
de la ciudad es alta pero los tres lugares presentan un buen aislamiento en relación a la contaminación lumínica de la ciudad. Promediando las observaciones de Tegucigalpa (tabla 4) para tener una magnitud límite global se observa lo siguiente:

Tabla 4: Estadística descriptiva de las magnitudes aparentes observadas desde Tegucigalpa

\begin{tabular}{ccc}
\hline Promedio & Mediana & Desviación estandar \\
\hline 3.53 & 3.56 & 0.41 \\
\hline
\end{tabular}

Observando los datos, se puede afirmar entonces, redondeando, que dentro de los límites de la ciudad la mejor magnitud observable es de una cuarta magnitud.

\section{Conclusiones}

- Se ha tomado la magnitud límite estelar para cinco ubicaciones, cuatro de ellas en Tegucigalpa y una quinta en Talanga. La magnitud límite estelar en el área rural, es superior a la de la zona urbana, o sea, Tegucigalpa. Las magnitudes límite en Tegucigalpa son de 2.98, 3.56, 3.58 y 3.76 en los lugares donde se tomaron los datos. La magnitud límite estelar en Talanga es de 4.68.

- No fue posible la toma de datos estacional. Las condiciones meteorológicas en Honduras solamente permiten observaciones de cielo en buenas u óptimas condiciones durante los meses de noviembre y enero. Si bien el cielo de marzo hasta cierto grado es despejado, las condiciones de bruma igualmente dificultan la observación.

- La magnitud promedio global para Tegucigalpa presenta un valor de 3.53 con una desviación estándar de la muestra de 0.41, y una mediana de 3.56. Comparando con el valor de hipótesis efectivamente se confirma que la magnitud límite estelar es significativamente menor a 4.0.

- Existe una diferencia marcada entre las magnitudes de una ubicación urbana respecto a una ubicación rural, tomando como referencia la magnitud promedio de 3.53 para Tegucigalpa y 4.68 para Talanga.

\section{Recomendaciones}

Hay factores adicionales que no fueron tomados en cuenta al inicio del estudio y que tienen relevancia para la estimación de la Magnitud Límite Estelar, particularmente en lo que se refiere a las condiciones de visibilidad. Se dio, por ejemplo, en el mes de marzo, condiciones favorables para la observación en lo que concierne a la nubosidad, pero la bruma de la época no permitía hacer observaciones óptimas. Habría que iniciar un nuevo estudio en el cual se analice las condiciones de visibilidad.

De igual manera convendría profundizar en un estudio acerca de la contaminación lumínica dentro de la ciudad. En el presente estudio se hicieron observaciones puntuales acerca del problema, sin profundizar en él. Pero ya dada una metodología de medición de la claridad de la atmósfera y habiendo comprobado que, efectivamente, la contaminación lumínica es un factor decisivo en la observación, habría que iniciar un estudio más profundo para obtener mayor cantidad de datos y resultados. 


\section{Referencias}

Makela, V. (2016). Estimating limiting magnitude. Recuperado de: http: / / obs . nineplanet s . org/lm/ rjm.html.

Mallén, M. A. (2010). Magnitud límite estelar. Recuperado de: http://www.astroerrante. com/superior-contenidos/superior-articulos/principal-articulo-observacion/ 53-magnitud-limite-estelar.html.

Mike Hankey, L. (2019). International meteor organization. Recuperado de: https://www . imo. net/ observations/methods/visual-observation/major/observation/.

Observatory, N. O. A. (2016). Globe at night. Recuperado de: http://www. globeatnight .org/.

Znojil, V. (1988). Gnomonický Atlas Brno 2000.0. WGN, Journal of the International Meteor Organization, 16(4):137-140. 